\title{
Potential Role of Natural Dietary Compounds in the Modulation of Metabolomic Fingerprints of Cancer
}

\author{
Narayanan $\mathrm{NK}^{1^{*}}$, Tiwari AK ${ }^{2}$, Kunimasa $\mathrm{K}^{3}$, Ashby Jr $\mathrm{CR}^{4}$ and Narayanan $\mathrm{B}^{1}$ \\ ${ }^{1}$ Department of Environmental Medicine, New York University School of Medicine, NY, USA \\ ${ }^{2}$ Department of Biomedical Sciences, Tuskegee University, Tuskegee, AL, USA \\ ${ }^{3}$ Cancer Chemotherapy Center, Japanese Foundation for Cancer Research, Japan \\ ${ }^{4}$ Department of Pharmaceutical Sciences, St. John's University, Queens, NY, USA
}

Metabolomics is a science of systems biology that allows global assessment of endogenous metabolites within a biological system. It represents a "snapshot" reading of gene function, enzyme activity, and the physiological landscape. Metabolite detection, either individually or grouped as metabolomics profile, is usually performed in cells, tissues, or biofluids. This is accomplished by either nuclear magnetic resonance spectroscopy or mass spectrometry, followed by multivariate data analysis. Since the loss of metabolic homeostasis is known to be involved in the pathogenesis of many diseases, the metabolome could have multiple applications, including identifications of biomarkers and drug target validation. Although there is evidence of successful preclinical applications, the clinical utility and application of metabolomics in diseases is just emerging.

Natural products, particularly those from medicinal plants, are one of the major dietary sources of compounds that have efficacy against cancer and other diseases. Our group and others have shown the clinical utility of some of the compounds derived from plants. For example, resveratrol, a stilbenoid derivative, found in the skin of red grapes and a constituent of red wine, has anti-aging properties and may be effective in preventing vascular diseases, cancers, viral infection or neurodegenerative processes [1]. Ellagic acid is a polyphenol primarily found in the seeds of the raspberry as ellagitannins, impairs carcinogenesis through inhibition of the cytochrome-P450 enzymes CYP 2A2, 3A1, 2C11,2B1, 2B2 and 2C6, by preventing the metabolism of certain compounds to carcinogens [2]. Curcumin, an active ingredient of turmeric (curry powder), has been linked with the prevention and treatment of a wide variety of cancers through modulation of multiple cell signaling pathways [3]. Omega-3 fatty acids from fish oil has been reported to 1) reduce cardiovascular morbidity and mortality; 2) increase the likelihood of healthy brain development in infants; 3 ) prevent certain forms of cancer; 4) promote optimal brain and vision function; 5) reduce the probability of arthritis, hypertension, diabetes mellitus and neurological/neuropsychiatric disorders [4]. Caffeic Acid Phenethyl Ester (CAPE), a major constituent of honeybee propolis, is a commonly used dietary supplement known to have anti-tumor, antiinflammatory, antioxidant, and immunomodulatory properties [5]. Melinjo (Gnetum gnemon L.) seeds are rich in stilbenoid dimers, such as Gnetin C (GC) and its glucosides (gnemonosides A, C, and D). Melinjo Seed Extracts (MSE) via antioxidant action, can 1) inhibit bacterial lipase; 2) stimulate amylase activity; 3) inhibit tyrosinase; 4) produce antiangiogenic, anti-tumor and anti-metabolic effects [6,7]. Recently, it has been shown that the combination of bioactive components or pharmaceutical compounds produce synergistic beneficial anticancer effects in various in vivo tumor models $[8,9]$. However, the molecular mechanism for their anticancer activity of bioactive components remains to be elucidated.

Currently, studies are being directed towards characterization and identification of the metabolic events involved in tumor growth and their reversal or blockage by natural compounds. We envision that this approach will help to (a) promote metabolic biomarker discovery for preventive or therapeutic approaches using natural compounds and (b) provide molecular insights for the development of novel anticancer treatments targeting tumor growth in altered metabolic pathways. We have conducted experiments using natural products focusing on metabolic targets in pancreatic cancer to provide evidence that natural compounds can alter cancer metabolomics. We hypothesized that KRAS-driven signals may initiate an abnormal chain of metabolic alterations, including enhanced glycolysis, diverted glutamine consumption, anomalous pentose phosphate pathway and autophagy. These aforementioned alterations may induce pancreatic cancer cells to become dependent upon certain metabolic pathways, thereby playing a major role in the development, survival and invasiveness of pancreatic tumor cells. To test our hypothesis, we performed microarray-based global gene expression profiling, using RNA isolated from cancer cells, in the presence and absence of MSE or GC to determine whether MSE or GC- induced anticancer activity by modulating targets associated with cancer metabolism. Mammalian Target of Rapamycin (mTOR), a central downstream target of Akt, is a highly conserved protein kinase, and is frequently activated in Pancreatic Ductal Adenocarcinoma (PDAC). mTOR, a master regulator of cell growth and proliferation downstream of oncogenic signaling pathways, controls specific aspects of cellular metabolism by inducing gene expression [10]. Our recent preliminary studies with plant-derived products, Melinjo Seed Extract (MSE) and its major component Gnetin C (GC), indicated that MSE and GC significantly inhibit human pancreatic cancer cells proliferation. Consistent with our in vitro findings, the dietary administration of MSE $(2 \% \mathrm{w} / \mathrm{w})$ significantly inhibited the formation of Pancreatic Intraepithelial Neoplasia (PanIN) and prevented the development of PDAC in LSL-Kras ${ }^{\mathrm{G} 12 \mathrm{D}} / \mathrm{p} 48$-Cre mice, a well-established mouse model of pancreatic cancer. However, the molecular mechanism by which MSE or GC prevent pancreatic cancer remains to be determined The regulation of transcription by the Mitogen-Activated Protein Kinase (MAPK) pathway has been reported to play a key role in metabolic reprogramming in PDAC. Studies also suggest that oncogenic constitutively active KRAS and its overactivated downstream signaling factors, such as B-Raf, phosphatidylinositol-3-kinase (PI3K) and Akt/ mTOR, are involved in metabolic pathways of pancreatic cancer [11].

*Corresponding author: Narayanan NK, Department of Environmental Medicine, New York University School of Medicine, New York, USA, Tel: 845-731-3625; E-mail: Narayanan.Narayanan@nyumc.org

Received December 26,2014 Accepted December 29, 2014; Published December 31, 2014

Citation: Narayanan NK, Tiwari AK, Kunimasa K, Ashby Jr CR, Narayanan B (2014) Potential Role of Natural Dietary Compounds in the Modulation of Metabolomic Fingerprints of Cancer. Metabolomics 5: e131. doi:10.4172/2153-0769.1000e131

Copyright: $\odot 2014$ Narayanan NK, et al. This is an open-access article distributed under the terms of the Creative Commons Attribution License, which permits unrestricted use, distribution, and reproduction in any medium, provided the original author and source are credited. 
Citation: Narayanan NK, Tiwari AK, Kunimasa K, Ashby Jr CR, Narayanan B (2014) Potential Role of Natural Dietary Compounds in the Modulation of Metabolomic Fingerprints of Cancer. Metabolomics 5: e131. doi:10.4172/2153-0769.1000e131

Page 2 of 2

Consistent with other studies, our preliminary microarray analysis indicated that MSE and GC modulate key targets in glucose and lipid metabolism, cholesterol biosynthesis and amino acid metabolism, as well as downregulating oncogenic KRAS and Akt/mTOR signaling pathway genes. Based on our preliminary findings from microarraybased gene expression profiling studies, we provide a summary of the effect of MSE on the oncogenic KRAS-driven signaling networks in tumor microenvironment potentially associated with pancreatic cancer metabolism (Figure 1). In summary, dietary substances with anti-tumor activities may have the potential to modulate the KRASdriven, oncogenic pathway-reprogrammed cancer cell metabolism and prevent the development of pancreatic cancer, as well as other cancers types, such as cancers of the colon and lung.

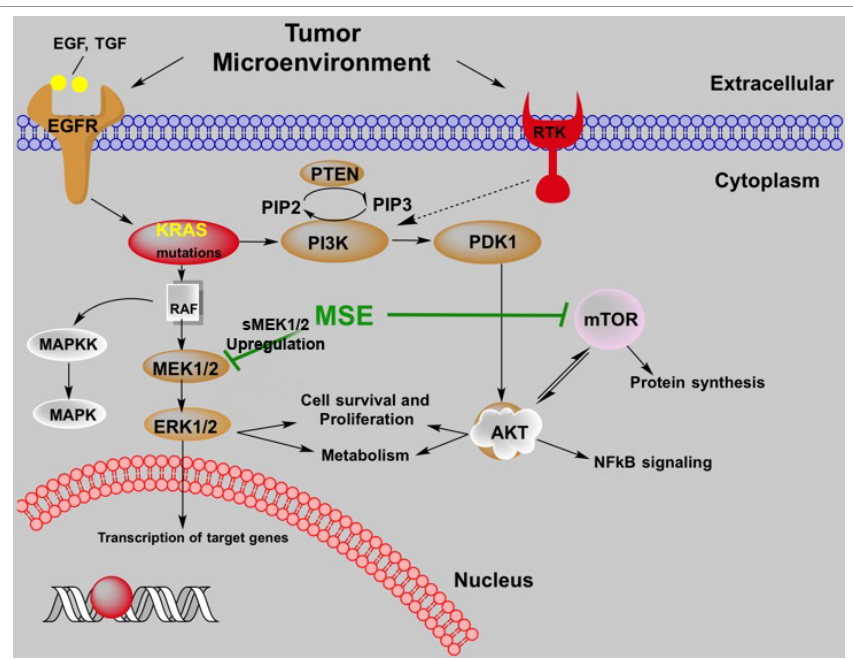

Figure 1: Schematic overview on the effect of dietary MSE on the KRASdriven oncogenic signaling network associated with cell survival and proliferation, including metabolism in pancreatic cancer. Membrane EGFR and other RTKs play a critical role in the development and progression of many types of cancer. Pro-tumorigenic inflammatory signaling induced in the tumor microenvironment activates KRAS by positive feedback mechanisms via EGFR and RTKs in an autocrine-paracrine manner. Mutated oncogenic KRAS interacts with the PI3K/AKT/mTOR signaling network and transform normal cells, leading to the initiation and progression of pancreatic cancer. Also, oncogenic KRAS signals through the Raf-MEK-ERK pathway and contributes to cancer development and progression. MSE may potentially inhibit these signaling pathways and preventing the formation of early neoplastic lesions and development of pancreatic cancer.

\section{References}

1. Gupta C, Prakash D (2014) Phytonutrients as therapeutic agents. J Complement Integr Med 11:151-169.

2. Celik G, Semiz A, Karakurt S, Arslan S, Adali O et al. (2013) A Comparative Study for the Evaluation of Two Doses of Ellagic Acid on Hepatic Drug Metabolizing and Antioxidant Enzymes in the Rat. Biomed Res Int 2013: 9

3. Hasima N, Aggarwal BB (2014) Targeting proteasomal pathways by dietary curcumin for cancer prevention and treatment. Curr Med Chem 21: 1583-1594.

4. Kaur N, Chugh V, Gupta AK (2014) Essential fatty acids as functional components of foods- a review. J Food Sci Technol 51:2289-2303.

5. Murtaza G, Karim S, Akram MR, Khan SA, Azhar S et al. (2014) Caffeic acid phenethyl ester and therapeutic potentials. Biomed Res Int 2014:9.

6. Kunimasa K, Ohta T, Tani H, Kato E, Eguchi R et al. (2011) Resveratro derivative-rich melinjo (Gnetum gnemon L.) seed extract suppresses multiple angiogenesis-related endothelial cell functions and tumor angiogenesis. Mol Nutr Food Res 55:1730-1734.

7. Tani H, Hikami S, lizuna S, Yoshimatsu M, Asama T et al. (2014) Pharmacokinetics and safety of resveratrol derivatives in humans after oral administration of melinjo (Gnetum gnemon L.) seed extract powder. J Agric Food Chem 62:1999-2007.

8. Narayanan NK, Nargi D, Randolph C, Narayanan BA (2009) Liposome encapsulation of curcumin and resveratrol in combination reduces prostate cancer incidence in PTEN knockout mice. Int J Cancer 125: 1-8.

9. Reddy BS, Patlolla JM, Simi B, Wang SH, Rao CV (2005) Prevention of colon cancer by low doses of celecoxib, a cyclooxygenase inhibitor, administered in diet rich in omega-3 polyunsaturated fatty acids. Cancer Res 65: 8022-8027.

10. Cornu M, Albert V, Hall MN (2013) mTOR in aging, metabolism, and cancer. Curr Opin Genet Dev 23:53-62.

11. Eser S, Schnieke A, Schneider G, Saur D (2014) Oncogenic KRAS signalling in pancreatic cancer. Br J Cancer 111:817-822. 